\title{
Plasma doping implant depth profile calculation based on ion energy distribution measurements*
}

\author{
L. Godet, ${ }^{\text {a) }}$ Z. Fang, S. Radovanov, S. Walther, and E. Arevalo \\ Varian Semiconductor Equipment Associates, 35 Dory Road, GL-17 Gloucester, Massachusetts 01930 \\ F. Lallement \\ STMicroelectronics, Crolles 38926, France \\ J. T. Scheuer and T. Miller \\ Varian Semiconductor Equipment Associates, 35 Dory Road, GL-17 Gloucester, Massachusetts 01930 \\ D. Lenoble \\ STMicroelectronics, Crolles 38926, France \\ G. Cartry and C. Cardinaud \\ Institut des Matériaux Jean Rouxel, CNRS-Nantes University, Nantes 44322, France
}

(Received 16 February 2006; accepted 16 August 2006; published 25 September 2006)

\begin{abstract}
In traditional beamline implantation, the incident ion mass and energy are well known parameters and simulation programs are available to predict the implant profiles. In plasma based ion implantation, all ionized species present in the plasma are extracted and implanted by applying negative voltage pulses to the wafer. Therefore, prediction of implant profile is more complicated since it requires the knowledge of relative abundance of each ion species as well as their energy distribution prior to entering the wafer surface. This information is not readily available using conventional plasma characterization techniques because most of them measure plasma bulk properties. In order to collect the information needed for predicting plasma implant profiles, an ion mass and energy spectrometer is installed at the wafer level to allow in situ measurement of ion mass and energy distribution. In this paper, $\mathrm{BF}_{3}$ plasma in the pressure range from 30 to $250 \mathrm{mTorr}$ is studied. The relative flux and energy distribution of $\mathrm{B}^{+}, \mathrm{BF}^{+}, \mathrm{BF}_{2}^{+}$, and $\mathrm{BF}_{3}^{+}$ions striking the wafer surface with energies up to $1 \mathrm{keV}$ are measured. As expected, no energy contamination was observed during a plasma doping implantation and the maximum energy of the ions is defined by the cathode voltage. Based on the spectrometer data, a series of simulations was performed to calculate the boron and fluorine dopant depth profiles. The calculated profiles were in good agreement with secondary ion mass spectrometry (SIMS) results and give some additional explanations of the unique surface-peaked SIMS profile of plasma doping implantation. () 2006 American Vacuum Society. [DOI: 10.1116/1.2353841]
\end{abstract}

\section{INTRODUCTION}

Transistor doping challenges at advanced technology nodes, as illustrated by the recent International Technology Roadmap for Semiconductors (ITRS), include high dose low energy implants for gate, source-drain, and source-drain extension dopings. ${ }^{1,2}$ At low implant energies, traditional beamline technology suffers from low beam transport efficiency due to space charge related difficulties. A beamline implanter, running in decel mode, can achieve higher beam current. Unfortunately, ions can undergo charge exchange collisions with residual gas creating fast neutrals. Unaffected by the decel lens, these fast neutrals reach the wafer with a higher energy than desired. The beamline implanter applications are limited by this energy contamination problem. ${ }^{3}$ The use of a cluster beam is another approach to improve low energy performance, though its production worthiness has not been completely demonstrated. ${ }^{4}$

\footnotetext{
*No proof corrections received from author prior to publication.

${ }^{\text {a) }}$ Author to whom correspondence should be addressed; electronic mail: ludovic.godet@vsea.com
}

Plasma doping is a very promising candidate for sourcedrain extension doping for the technology node below $65 \mathrm{~nm}$, as it can provide low-energy implantation and very high doses. It allows for less integration steps (no offset spacers) and better or equal electrical performance as compared to traditional ion implantation. ${ }^{5}$ Plasma doping has been developed to meet the low energy high dose implant needs. ${ }^{5}$ It has been demonstrated that plasma doping (PLAD) profiles are shallow, abrupt, and surface peaked over the energy range of $0.05-10 \mathrm{kV} .{ }^{6,7}$ A PLAD implant profile is determined not only by the implant energy and dose, as in the beamline case, but also by parameters such as plasma density and composition. The principle and detailed description of PLAD can be found elsewhere, ${ }^{8}$ and only a brief introduction is given here.

The glow discharge PLAD chamber consists of an anode and a cathode, which holds the wafer being implanted. A negative dc voltage pulse is applied to the cathode, which generates a glow discharge in the volume adjacent to the wafer. The plasma is ignited by each voltage pulse applied to the wafer, and at the pulse end, plasma follows a natural 
decay process. During the pulse-on period, ions in the plasma are accelerated by the electric field across the plasma sheath and are subsequently implanted into the wafer. The energy of ions striking the wafer during pulse-on period is mainly controlled by the applied voltage. Other parameters such as pulse width, frequency, gas pressure, flow rate, surface condition, and geometry of electrodes can also affect the implant process by changing plasma composition, ion flux, and the number of collision inside the sheath. One parameter of particular interest to plasma doping is gas pressure. This parameter affects collisions between ions and neutrals in the sheath, which can alter the ion's energy. In this paper, the gas pressure effect is examined in detail and its impact to dopant profile is fully characterized in the sub-kilovolt range.

We use the computer simulation package Stopping and Range of Ions in Matter ${ }^{9,10}$ (SRIM) to predict dopant profiles of all species and energies allowed by the PLAD voltage. The plasma implant profile is constructed from the individual profiles at different energies with their contributions proportional to the relative amount of ions at these energies. The essential information for profile calculation are the ion mass and energy distribution, which are measured in situ in the center of the wafer using an ion mass and energy spectrometer. Due to the pulsed plasma nature, the ion energy distribution of the ions striking the cathode changes over time during the plasma development and decay, therefore time resolved measurements are necessary. In this particular paper, the effect of the discharge pressure on the ion energy distribution (IED) of the ions striking the wafer during the pulse-on period is studied using the time resolved operation mode of the mass spectrometer.

\section{EXPERIMENTS}

Figure 1 shows a schematic drawing of the PLAD chamber, where implants and plasma diagnostic experiments were carried out. The pulsed plasma is produced between two parallel electrodes: a grounded anode and a negatively biased cathode on which the silicon wafer is loaded. An electrostatic quadrupole probe (EQP) analyzer from Hiden Analytical Inc. is installed below the wafer. A small aperture at the center of the wafer allows ions to enter the analyzer.

The Hiden EQP system consists of an electrostatic ion energy analyzer followed by a quadrupole mass spectrometer. It is electrically isolated from the cathode by a differentially pumped vacuum gap. A high vacuum level $(<5$ $\times 10^{-8}$ Torr) is maintained to eliminate any collisions inside the EQP system. The aperture size on the $\mathrm{Si}$ wafer is $100 \mu \mathrm{m}$, which is selected according to the chamber pressure and vacuum level required inside EQP. The use of a Si wafer with a small aperture also eliminates any metal contamination risk inside the process chamber. Modifications to the extraction sector of the analyzer are made to achieve high ion transmission efficiency $(>95 \%)$ over the energy range $(0-1000 \mathrm{eV}){ }^{11}$

For time resolved measurements, the EQP analyzer is gated by a transistor-transistor logic (TTL) pulse, $0-5 \mathrm{~V}$, that is synchronized with the PLAD voltage pulse. A $2 \mu \mathrm{s}$

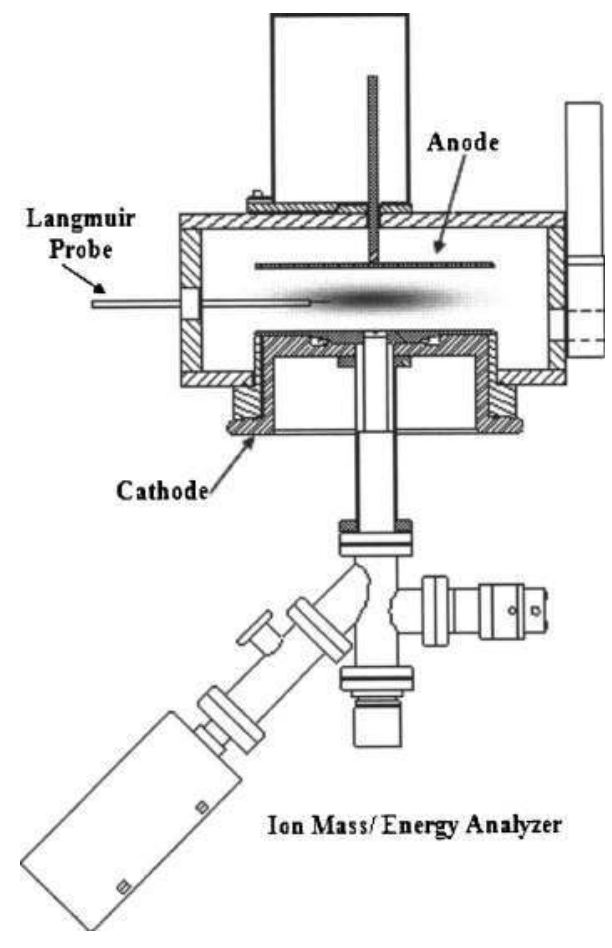

FIG. 1. Schematic diagram of PLAD process chamber with ion mass/energy analyzer and a Langmuir probe.

time resolution is achievable with the current EQP probe. The delay time between the TTL pulse and PLAD pulse is adjustable to allow ion sampling at the beginning, middle, and end of the PLAD pulse. The TTL pulse width is optimized taking into account the trade-off between good time resolution and low count rate. We measure ion energy from 0 to $1000 \mathrm{eV}$. In the steady state, the energy of each ion species is well known and the mass spectrometer time resolution is adequate. During the rise and fall times of the pulse, the time resolution is insufficient to accurately determine the shape of the IED. However, during these dynamic changes, the relative ion flux measured remains valid. In this paper, the steady state ion energy distributions are normalized to the relative total ion flux, defined as the sum of the ion energy distribution of all the ions reaching the cathode.

To collect PLAD dopant profiles, $n$-type prime $\mathrm{Si}$ (100) wafers with $12 \AA$ of control thermal oxide were implanted using PLAD with different plasma parameters. For this study, $0.5 \mathrm{kV}$ and $1000 \mathrm{~V}$ PLAD pulses with $50 \mu$ s pulse-on time and $2.5 \mathrm{kHz}$ frequency (12.5\% duty cycle) were applied to the wafer. The $\mathrm{BF}_{3}$ pressure in the process chamber ranged from 30 to $70 \mathrm{mTorr}$ for $1 \mathrm{kV}$ PLAD and from 75 to 250 mTorr for $0.5 \mathrm{kV}$ PLAD. The as-implanted boron profile was analyzed using secondary ion mass spectrometry (SIMS) with $700 \mathrm{eV} \mathrm{O}+2$ at $45^{\circ}$ incidence angle with $\mathrm{O}_{2}$ leak. As the coimplanted fluorine plays an important role during the activation of dopant, ${ }^{12}$ as-implanted fluorine profile was also collected using SIMS with $500 \mathrm{eV} \mathrm{Cs}^{+}$at $60^{\circ}$ incidence angle without $\mathrm{O}_{2}$ leak. The dose errors for all measurements are expected to be less than $20 \%$. The absolute error in the junction depth, $X j$, should be less than $10 \%$. This absolute 


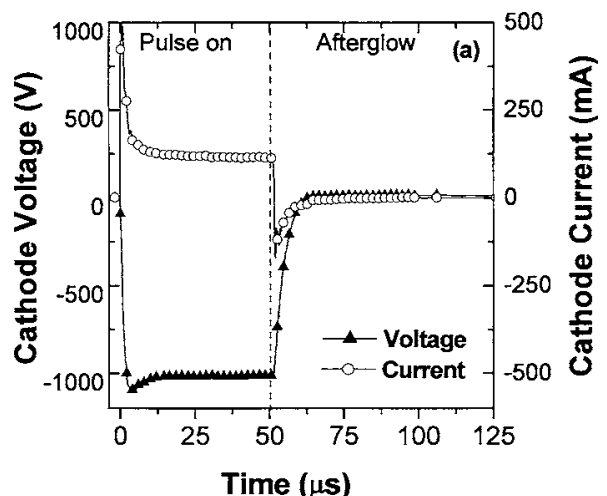

FIG. 2. Cathode voltage and current wave forms for a $1 \mathrm{kV}, 40 \mathrm{mTorr}$, $50 \mu \mathrm{s}, 2.5 \mathrm{kHz} \mathrm{BF}$ discharge.

error is mainly due to discrepancies among techniques (e.g., nuclear reaction analysis, Rutherford backscattering spectrometry, etc.) and laboratories which are used to calibrate the SIMS equipments. ${ }^{13}$ These conditions allow accurate measurement of profile shape, but less accurate measurement of total dose.

\section{RESULTS AND DISCUSSION}

In Fig. 2, we show the voltage and current wave forms measured during a $1 \mathrm{kV}, 40 \mathrm{mTorr} \mathrm{BF}_{3}$ discharge. The current pulse has a sharp peak at the leading edge, which is mainly due to displacement current. ${ }^{14}$ As soon as the voltage pulse is applied, a dynamic process starts in the discharge chamber where the neutrals and charged particles adjust their quantity, position, and energy until a steady state is reached. Characterization of the dynamic process is difficult. ${ }^{15} \mathrm{~A}$ concern associated with pulse rise and fall times is that it may affect the ion mass and energy distribution, thus changing the implant profile. This concern was ruled out in early studies on low energy $\mathrm{BF}_{3}$ PLAD implantation, as boron SIMS profiles showed no difference for different pulse-on widths ${ }^{16}$ $(20-60 \mu \mathrm{s})$ with constant rise and decay times. The IEDs are changing during the rise and fall times of the pulse-on period. During these dynamic changes, the ion density and the ion energy are lower than during the flat part of the pulse. In Fig. 3, we report the time evolution of the different ion fluxes measured with the mass spectrometer during the PLAD pulse. During the first $10 \mu$ s of the pulse-on period, the sheath expands and the relative ion flux increases. After $15 \mu \mathrm{s}$, the relative total ion flux is stabilized and the IEDs of the different ions remain the same. During the decay, the total ion flux decreases as the sheath collapses. This observation allows us to improve the statistics of the EQP system by employing a wide TTL gate pulse starting at $20 \mu$ s after the beginning of the pulse and ending at $50 \mu \mathrm{s}$. Therefore, only the constant portion of the pulse will be used to calculate the dopant depth profile. The rise and fall parts of the voltage pulse will not be included in the measurement and calculation as they do not contain any critical information for implant profile calculation.

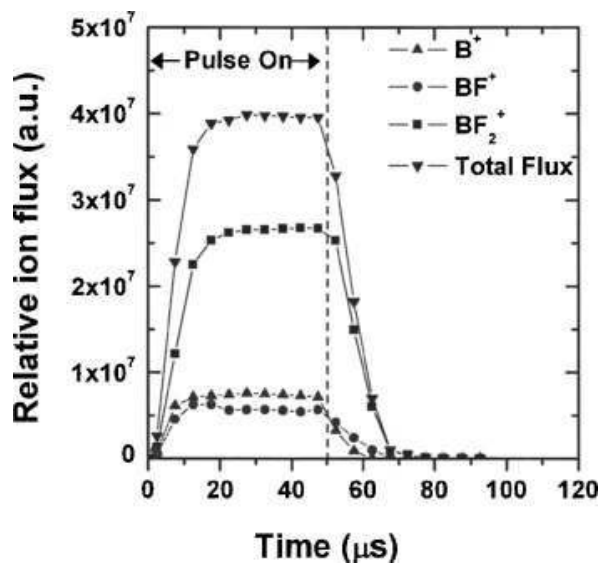

FIG. 3. Evolution during the pulse of the relative total ion flux and $\mathrm{B}^{+}, \mathrm{BF}^{+}$, and $\mathrm{BF}_{2}^{+}$ion relative fluxes measured by time resolved mass spectrometry.

In order to understand the shape of the energy distribution of different ion species reaching the cathode, a good understanding of the sheath dynamics is necessary. In all the experiments reported here, the discharge is operated at relatively higher pressures where the collision mean free path $(\lambda)$ is short, so that many collisions occur within the sheath of thickness $s$. Unfortunately, certain approximation has to be used to describe the ion-neutral interaction because ionneutral cross sections for a $\mathrm{BF}_{3}$ discharge are not well known. The hard sphere model is used here to estimate the ion-neutral cross section for a $\mathrm{BF}_{2}^{+}$ion. In this paper, a constant ion-neutral cross section of $2 \times 10^{-15} \mathrm{~cm}^{2}$ will be assumed for different pressures and cathode voltages. The number of collisions $(s / \lambda)$ inside a sheath is calculated based on the collisional Child law ${ }^{17-19}$ by using the formula (1). The assumptions are that the ion motion is highly collisional and charge exchange is the dominant ion-neutral collision mechanism; therefore the sheath thickness is significantly larger than the mean free path,

$$
\frac{s}{\lambda}=\left[\left(\frac{2}{3}\right)\left(\frac{5}{3}\right)^{3 / 2} \varepsilon_{0}\left(\frac{2 e}{\pi M}\right)^{1 / 2}\left(\frac{\sigma}{k T_{g}}\right)^{2} \frac{V_{0}^{3 / 2} p^{2}}{j_{i}}\right]^{2 / 5},
$$

where $\varepsilon_{0}$ is the permittivity of free space, $e$ is the charge of an ion of mass $M, \sigma$ is the ion-neutral cross section, $k$ is Boltzmann's constant, $T_{g}$ is the gas temperature, $V_{0}$ is the cathode voltage, $p$ is the neutral-gas pressure, and $j_{i}$ is the ion current density. The ion current density is calculated using the following formula:

$$
j_{i}=\frac{I_{c}}{a_{c}(1+\gamma)},
$$

where $I_{c}$ is the measured cathode current, $a_{c}$ is the cathode area, and $\gamma$ is the secondary electron emission coefficient which was experimentally determined for our apparatus. ${ }^{20} \mathrm{In}$ these experiments, the secondary emission current is a significant fraction of the measured cathode current and is taken into account during the calculation.

In Fig. 4, we show the number of collisions inside the sheath calculated with relation (1) for different gas pressures 


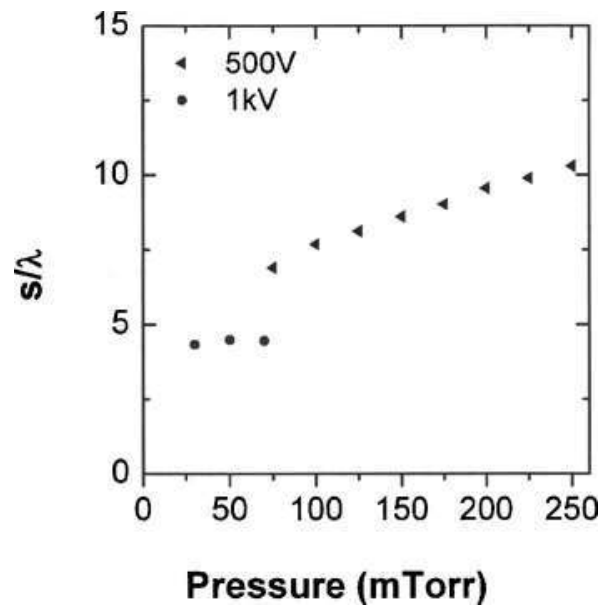

FIG. 4. Ratio of sheath thickness over ion mean free path $s / \lambda$ as a function of the pressure for different cathode voltage $(1 \mathrm{kV}$ and $500 \mathrm{~V}) . s / \lambda$ is an estimation of the number of collisions for an ion crossing the high voltage sheath in front of the cathode.

and two extreme cathode voltages, 500 and $1000 \mathrm{~V}$ (discharge cannot be ignited below $500 \mathrm{~V}$, and due to the limitation of the mass spectrometer to ion energy below $1000 \mathrm{eV}, 1000 \mathrm{~V}$ corresponds to the maximum energy that we can measure). When the cathode bias voltage is at $1 \mathrm{kV}$, the discharge pressure is limited by the Paschen curve to a range from 30 to $70 \mathrm{mTorr}$. For this pressure range the number of collisions inside the sheath remains constant $(s / \lambda$ remains constant). The decrease of the mean free path at higher pressure is compensated by the decrease of the sheath thickness due to plasma density increase at higher pressure. Consequently, minor changes in the shape of the IED are expected. When a lower bias voltage is used $(0.5 \mathrm{kV})$, the discharge can be operated under a large range of pressures from 75 to 250 mTorr. Within this pressure range, the number of collision increases from 6.9 to 10.3 , thus causing significant changes in the IED. This is in agreement with our experimental observations.

In Fig. 5 we show the cathode current for different rela-

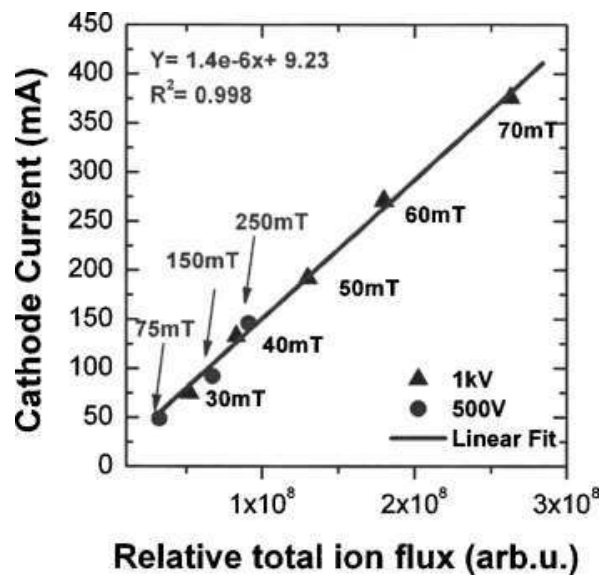

FIG. 5. Cathode current vs relative total ion flux measured with the mass spectrometer for $1 \mathrm{kV}$ (30-70 mTorr) and $500 \mathrm{~V}$ (75-250 mTorr) $\mathrm{BF}_{3}$ plasma.

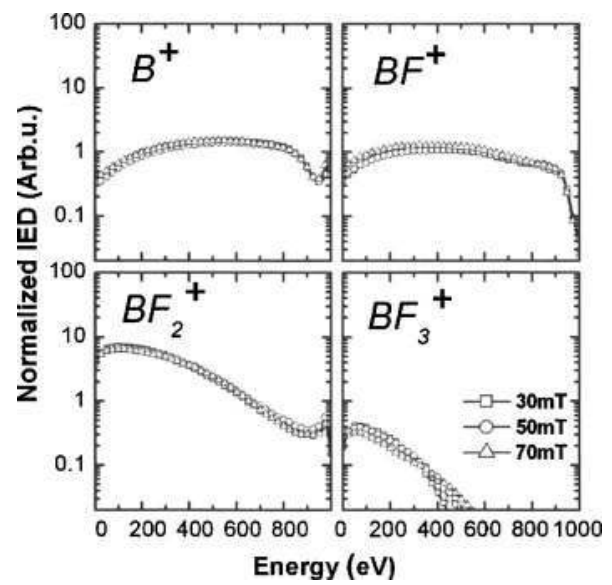

FIG. 6. $\mathrm{B}^{+}, \mathrm{BF}^{+}, \mathrm{BF}_{2}^{+}$, and $\mathrm{BF}_{3}^{+}$IED normalized to the total ion flux for $1 \mathrm{kV}$ $\mathrm{BF}_{3}$ plasma at three different pressures: 30,50 , and $70 \mathrm{mTorr}$.

tive ion fluxes for the $1 \mathrm{kV}$ bias voltage. The cathode current is increasing rapidly from $75 \mathrm{~mA}$ at $30 \mathrm{mTorr}$ to $375 \mathrm{~mA}$ at 70 mTorr. At the same time the total relative ion flux increases from $5.2 \times 10^{7}$ to $2.6 \times 10^{8}$ in unit of counts/s. A linear relationship is observed between cathode current and relative total ion flux. In the case of the $0.5 \mathrm{kV} \mathrm{BF}_{3}$ discharge, the linear relationship is still valid over the large range of pressures. As the secondary emission yield $(\gamma)$ is almost constant with pressure, the cathode current is proportional to the ion current [relation (2)] and thus to the ion flux (ion flux is equal to ion current times elementary charge). The linear relation between ion flux measured by the mass spectrometer and the cathode current suggests a constant transmission inside the mass spectrometer in this pressure range.

The ion mass and energy distribution of a $1 \mathrm{kV} \mathrm{BF}$ plasma at three different pressures is presented in Fig. 6. As discussed previously, the pressure changes do not affect significantly the shape of the IED except for the $\mathrm{BF}_{3}^{+}$ion. In all cases, $\mathrm{BF}_{3}^{+}$fraction remains very low (less than $2 \%$ ). $\mathrm{BF}_{3}^{+}$has a smaller energy spread and the peak of the IED is in a thermal range. $\mathrm{BF}_{2}^{+}$is the most abundant ion implanted into the wafer with more than $60 \%$ of the total boron ion flux composed of $\mathrm{B}^{+}, \mathrm{BF}^{+}, \mathrm{BF}_{2}^{+}$, and $\mathrm{BF}_{3}^{+}$. Only a few ions $(<1 \%)$ from the boron ion flux are able to reach the wafer with full energy $(1000 \mathrm{eV})$. The majority of the ions strike the cathode with energy lower than $500 \mathrm{eV}$. Due to the collisions inside the sheath, typical ion energy distributions have a large energy spread producing shallow and more abrupt as-implanted SIMS profile than an equivalent $1000 \mathrm{eV}$ beamline implant, where all the ions strike the wafer with the full energy.

The normalized IED of $0.5 \mathrm{kV} \mathrm{BF} 3$ discharge in a range of pressure (100-250 mTorr) is shown in Fig. 7. The maximum energy of $\mathrm{B}^{+}, \mathrm{BF}^{+}$, and $\mathrm{BF}_{2}^{+}$is reduced significantly when the pressure is raised from 100 to 250 mTorr. Compared to the $1 \mathrm{kV}$ case, the number of collision inside the sheath is significantly increased. Only few ions $(<0.1 \%)$ from the boron ion flux are able to reach the wafer with full 


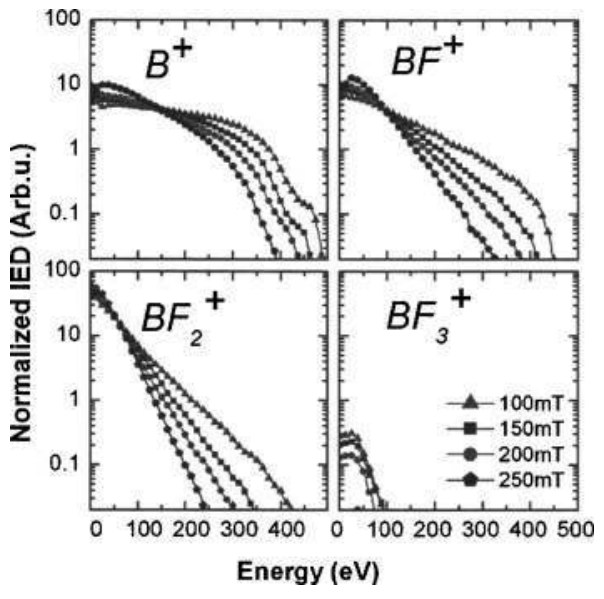

FIG. 7. $\mathrm{B}^{+}, \mathrm{BF}^{+}, \mathrm{BF}_{2}^{+}$, and $\mathrm{BF}_{3}^{+}$IED normalized to the total ion flux for $500 \mathrm{kV} \mathrm{BF} \mathrm{BF}_{3}$ plasma at four different pressures: 100, 150, 200, and 250 mTorr.

energy $(500 \mathrm{eV})$. The majority of these ions $(91 \%)$ strike the cathode with energy lower than $250 \mathrm{eV}$. The ion energy and the lighter ion fraction slightly decrease with the pressure, which should provide shallower dopant depth profile as confirmed by previous experiments. ${ }^{16}$ Figure 8 shows that $\mathrm{BF}_{2}^{+}$is the dominant ion reaching the cathode for all the different process conditions and the fraction of $\mathrm{BF}_{3}^{+}$is lower than $1 \%$ of total ion flux. The increase of collisions inside the sheath does not significantly affect the ratio between the different ions.

\section{CALCULATION OF THE PREDICTED DOPANT DEPTH PROFILE}

The measured IED for all the boron and fluorine ions implanted into the wafer was used as an input into an implant simulation software. The SRIM was used to simulate and predict an implant profile and the results were compared to the profiles measured by SIMS. ${ }^{21}$ Since SRIM only accepts atomic ions, the $\mathrm{BF}_{x}^{+}(x=1,2,3)$ IEDs are converted into

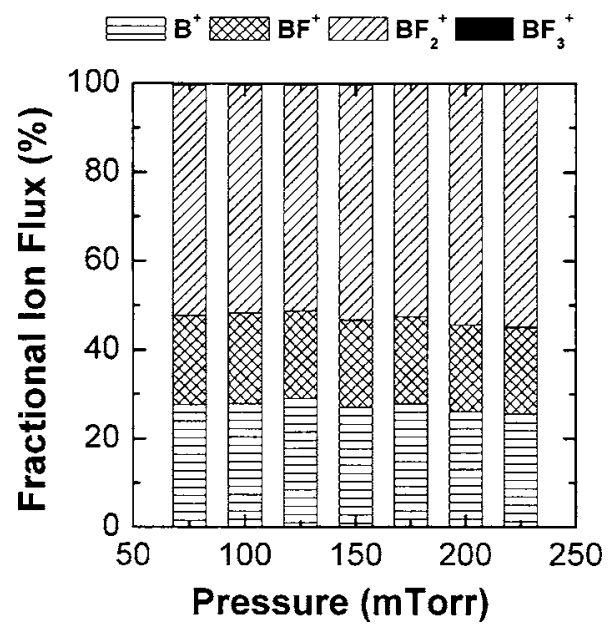

FIG. 8. Ion ratio in a $500 \mathrm{~V} \mathrm{BF}_{3}$ plasma with pressure ranging from 75 to 225 mTorr.
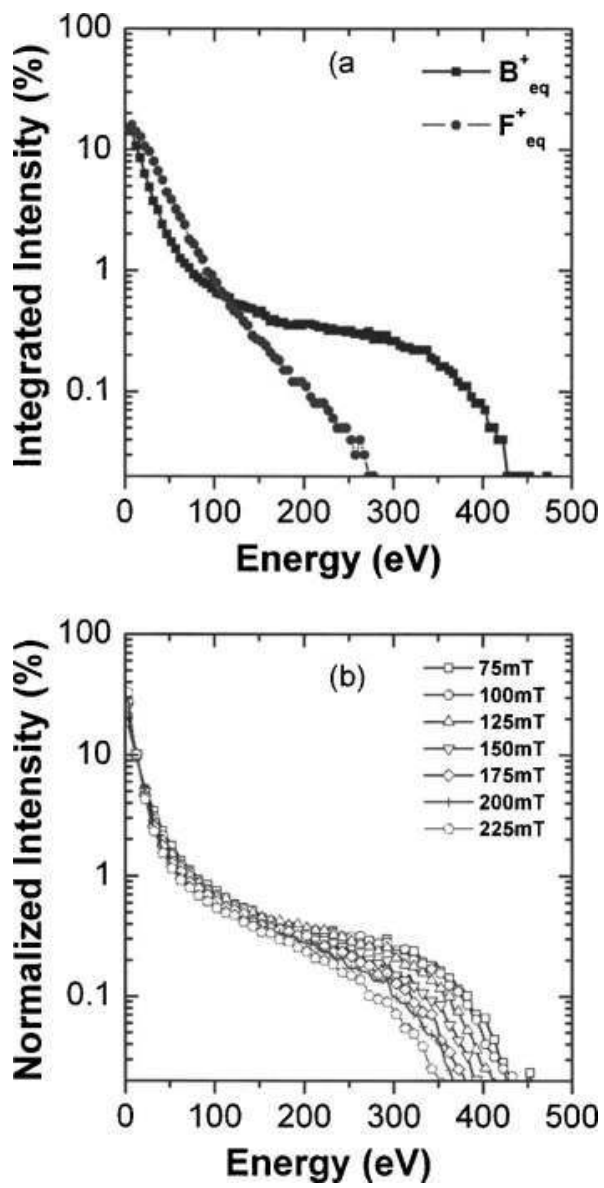

FIG. 9. (a) Boron and fluorine equivalent energies based on the $500 \mathrm{~V}$, 100 mTorr IED of the ions reaching the wafer. (b) Boron equivalent energy based on the $500 \mathrm{~V}$ IED of the ions reaching the wafer for a large range of pressure (75-250 mTorr).

equivalent boron IED $\mathrm{B}_{\mathrm{eq}}^{+}$and equivalent fluorine IEDs $\mathrm{F}_{\mathrm{eq}}^{+}$ by using the following formulas:

$$
\begin{aligned}
& E\left(\mathrm{~B}_{\mathrm{eq}}^{+}\right)=E\left(\mathrm{BF}_{x}^{+}\right) \frac{m_{\mathrm{B}}}{\mathrm{m}_{\mathrm{BF}_{x}^{+}}}, \\
& E\left(\mathrm{~F}_{\mathrm{eq}}^{+}\right)=E\left(\mathrm{BF}_{x}^{+}\right) \frac{m_{\mathrm{F}}}{m_{\mathrm{BF}_{x}^{+}}} .
\end{aligned}
$$

Figure 9(a) shows the equivalent boron and fluorine IEDs under the previous discharge conditions. As expected, the majority of the boron and fluorine equivalent ion energies are below $250 \mathrm{eV}$. Less energetic equivalent boron is obtained for higher pressure, but the low energy tail is not significantly affected [Fig. 9(b)]. The equivalent boron and fluorine energies are partitioned into energy bins with $10 \mathrm{eV}$ increments. Each energy bin defines an ion dose fraction of the total ion flux coming onto the wafer. The average energy of each bin is used as the input energy for the SRIM simulation. Then all the output depth profiles from SRIM are added up to represent the predicted depth profile based on the IED of all the ions striking the wafer. The predicted boron and fluorine profiles are compared to the experimental boron and fluorine 

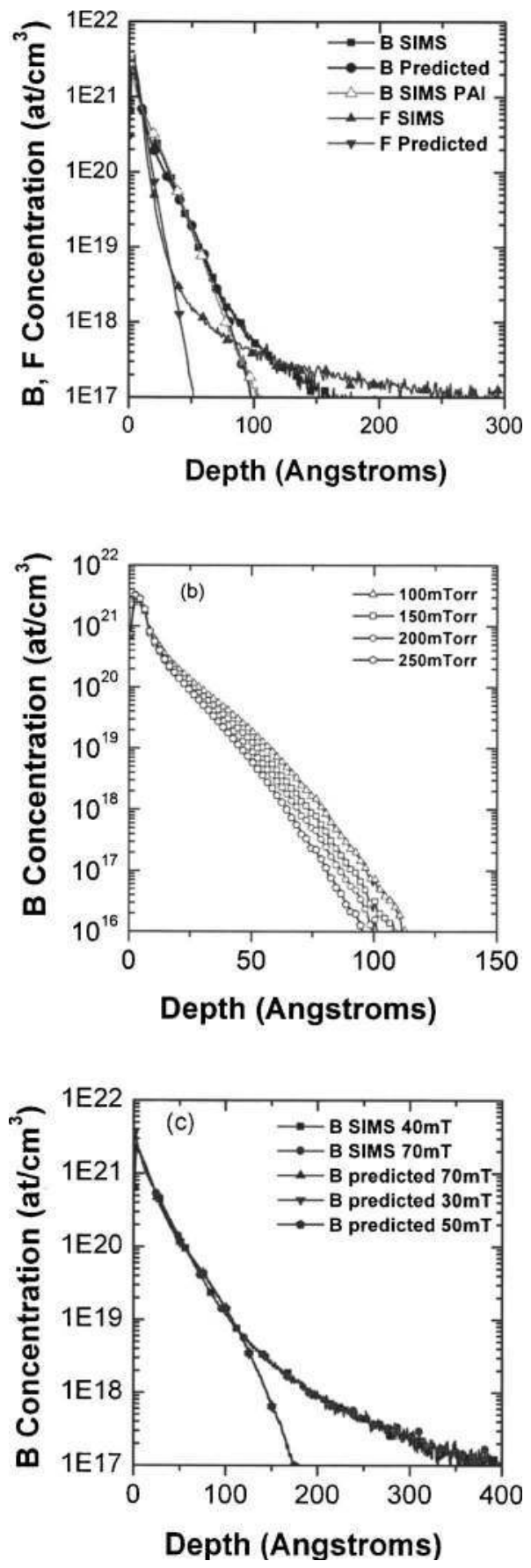

Fig. 10. (a) Boron and fluorine predicted depth profiles based on the measured ion mass and energy distribution for crystalline and preamorphized silicon wafer (PAI), compared with the measured SIMS depth profile for the same implant conditions ( $500 \mathrm{~V}, 100 \mathrm{mTorr}^{\mathrm{BF}_{3}}$ PLAD). (b) Boron predicted profile of $500 \mathrm{~V} \mathrm{BF}_{3}$ PLAD at 100, 150, 200, and 250 mTorr. (c) Boron predicted depth profile based on the measured ion mass and energy distribution, compared with the measured SIMS depth profile for the same implant conditions $\left(1 \mathrm{kV}, 30-70 \mathrm{mTorr}^{\mathrm{BF}} \mathrm{F}_{3} \mathrm{PLAD}\right)$.

SIMS profiles on Fig. 10(a). A good agreement is obtained between the simulation and the experimental SIMS profiles for both elements. On the boron and fluorine SIMS profiles, we clearly see the typical channeling tail ${ }^{22}$ inside the crystalline wafer. This was not reflected in the predicted profiles because SRIM simulation assumes an amorphous target. This
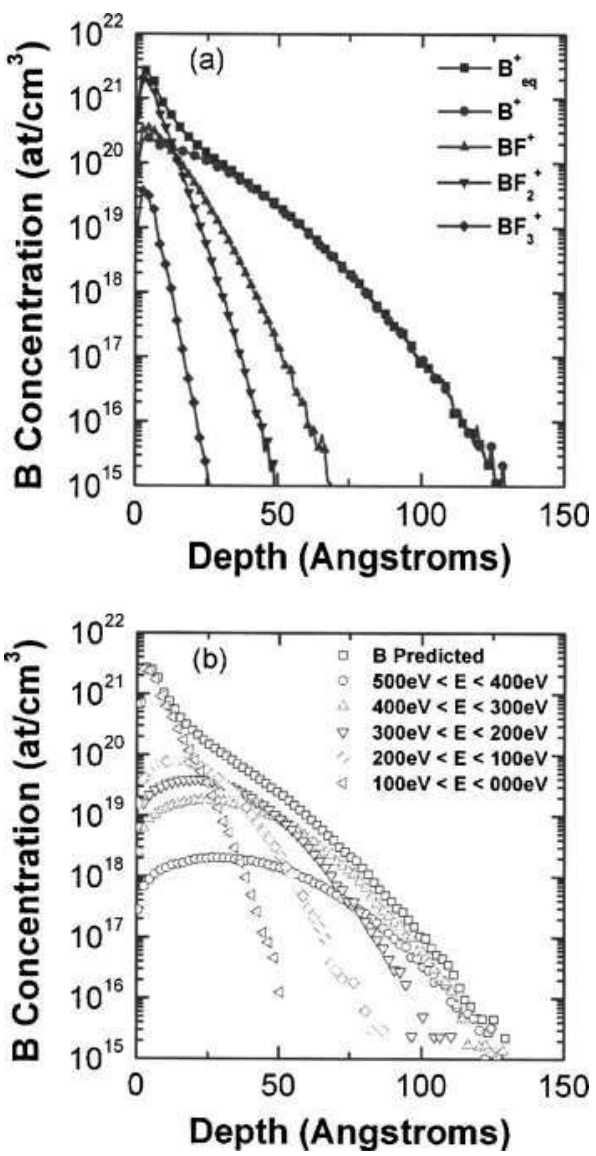

FIG. 11. (a) Contribution of each ion reaching the wafer $\left(\mathrm{B}^{+}, \mathrm{BF}^{+}, \mathrm{BF}_{2}^{+}\right.$, and $\mathrm{BF}_{3}^{+}$) onto the boron depth profile. (b) Contribution of each energy bin (0-100 eV, 100-200 eV, 200-300 eV, 300-400 eV, and 400-500 eV) equivalent boron energy onto the boron depth profile.

channeling tail, obtained at 0.5 and $1 \mathrm{kV}$ with a crystalline silicon wafer, confirmed that the ions reach the wafer with a small angle. When a preamorphized implanted (PAI) substrate is used, no channeling tail is observed and the predicted boron profile matches also the deeper part of the measured SIMS profile for the $0.5 \mathrm{kV}$ case [see "B SIMS PAI" curve on Fig. 10(a)]. As expected, based on the boron equivalent energy distribution, shallower boron profiles are obtained when the pressure increases from 100 to $250 \mathrm{mTorr}$ [see Fig. 10(b)]. No difference on the boron SIMS profile was observed when the pressure was raised from 30 to 70 mTorr as expected with the $1 \mathrm{kV}$ mass spectrometer data and the predicted profiles shown in Fig. 10(c). By doing the simulation for each ion separately for the $0.5 \mathrm{kV}$, 100 mTorr PLAD, the particularities of a PLAD dopant profile (surface peak and very shallow profile) can be explained. Figure 11(a) shows the role of each ion species reaching the wafer; $\mathrm{B}^{+}$defines the depth of the implant, $\mathrm{BF}^{+}$and $\mathrm{BF}_{2}^{+}$are the main contributors to the surface dose, and $\mathrm{BF}_{3}^{+}$does not have significant impact on the SIMS profile. Thus, in order to meet the junction depth requirements of the ITRS for technological nodes below $65 \mathrm{~nm}$, the $\mathrm{B}^{+}$ion flux fraction needs to be minimized by controlling the plasma parameters (bulk plasma optimization) and the collision processes inside the 
cathode sheath. This subject will be discussed in another paper (see also Ref. 11). It is also found that ions with equivalent energy between 0 and $100 \mathrm{eV}$ are the main contributors to the dose and the surface peak of a PLAD SIMS profile which represents about $50 \%$ of the total dose [Fig. 11(b)]. The higher energy ions are found to define the final dopant depth. Therefore, another approach to reduce the junction depth could be to increase further the number of collision and reduce the $\mathrm{B}^{+}$mean energy rather than trying to reduce the $\mathrm{B}^{+}$fraction.

\section{SUMMARY AND CONCLUSION}

In situ mass and ion energy distribution data were collected with an ion mass and energy spectrometer installed in the middle of the cathode. These data were used to predict the plasma implant profiles of a $\mathrm{BF}_{3}$ plasma based ion implantation. Plasma in the pressure range from 30 to 250 mTorr, as well as the relative flux and energy distribution of $\mathrm{B}^{+}, \mathrm{BF}^{+}, \mathrm{BF}_{2}^{+}$, and $\mathrm{BF}_{3}^{+}$ions striking the wafer surface with energies up to $1 \mathrm{kV}$, was studied in this paper. Based on the spectrometer data, a series of SRIM simulations was performed to calculate the boron and fluorine dopant depth profiles. The calculated profiles match the ones measured by secondary ion mass spectrometry (SIMS) and give some additional explanations of the surface-peaked SIMS profile unique to plasma doping. Such knowledge will be useful in controlling and optimizing implant profiles and dopant activation.

\footnotetext{
${ }^{1}$ International Technology Roadmap for Semiconductors $\rightarrow$ Front End Processes, Semiconductor Industry Association, p. 23, 2005, http:// www.itrs.net/Common/2005ITRS/FEP2005.pdf
}

${ }^{2}$ John O. Borland, Tomoko Matsuda, and Keiji Sakamoto, Solid State Technology, pp. 83-94, June 2002

${ }^{3}$ A. Renau and J. T. Scheuer, Proceedings of the 14th International Conference on Ion Implantation Technology, Taos, September 2002 (unpublished), p. 151.

${ }^{4}$ Z. Fang, E. Arevalo, Tim Miller, Harold Persing, Edmond Winter, and V. Singh, Proceedings of IWJT 2004 (unpublished).

${ }^{5}$ F. Lallement et al., Symp. VLSI Tech. Dig. 2004, 178.

${ }^{6}$ J. T. Scheuer et al., Surf. Coat. Technol. 186, 57 (2004).

${ }^{7}$ S. B. Felch, D. Lenoble, A. Grouillet, E. Arevalo, S. R. Walther, Z. Fang, B.-W. Koo, and R. B. Liebert, Proceedings of the 13th International Conference on Ion Implantation Technology, Alpbach, Austria, September 2000 (unpublished)

${ }^{8}$ B.-W. Koo, Z. Fang, L. Godet, S. Radovanov, C. Cardinaud, G. Cartry, A. Grouillet, and D. Lenoble, IEEE Trans. Plasma Sci. 32 (2004).

${ }^{9}$ Stopping and Range of Ions in Matter (SRIM), a numerical simulation by J. F. Ziegler and J. P. Biersack, http://www.srim.org/

${ }^{10}$ J. F. Ziegler, Ion Implantation: Science and Technology, edited by J. F. Ziegler (Ion Implantation Technology, Edgewater, MD, 1996).

${ }^{11}$ Ludovic Godet, Ph.D. thesis, Nantes University, 2006.

${ }^{12}$ E. Ishida, D. F. Downey, K. S. Jones, and J. Lui, Proceedings of the 1998 International Conference on Ion Implantation Technology (IEEE, New York, 1999), Vol. 2, pp. 909-912.

${ }^{13}$ T. H. Buyuklimanli, C. W. Magee, and J. W. Marino, J. Vac. Sci. Technol. B 24, 408 (2006).

${ }^{14}$ Z. Fang, Bon-Woong Koo, Susan Felch, Yu Lei, Lawrence J. Overzet, and Matthew Goeckner, Proceedings of the 14th International Conference on Ion Implantation Technology, Taos, September 2002 (unpublished), p. 403.

${ }^{15}$ S. Radovanov et al., J. Appl. Phys. 98, 113307 (2005).

${ }^{16} \mathrm{D}$. Lenoble, Ph.D. thesis, Toulouse University, 2000

${ }^{17}$ M. A. Lieberman, J. Appl. Phys. 86, 2926 (1989).

${ }^{18}$ R. A. Stewart and M. Lieberman, J. Appl. Phys. 70, 3491 (1991).

${ }^{19}$ Casper V. Budtz-Jorgensen, Ph.D. thesis, Faculty of Science, Aarhus University, 2001.

${ }^{20}$ Timothy Miller (unpublished).

${ }^{21}$ Steve Walther, Ludovic Godet, T. Buyuklimanli, and J. Weeman, J. Vac. Sci. Technol. B 24, 489 (2006).

${ }^{22}$ Steve Walther and Reuel Liebert, J. Vac. Sci. Technol. B 24, 482 (2006). 